\title{
Molecular analysis of the arylsulphatase A gene in late infantile metachromatic leucodystrophy patients and healthy subjects from Italy
}

\author{
Stefano Regis, Mirella Filocamo, Marina Stroppiano, Fabio Corsolini, Rosanna Gatti
}

\author{
Laboratorio di \\ Diagnosi Pre e Post \\ Natale di Malattie \\ Metaboliche, \\ Istituto G Gaslini, \\ Largo G Gaslini 5 , \\ 16147 Genova, \\ Italy \\ $S$ Regis \\ M Filocamo \\ M Stroppiano \\ F Corsolini \\ R Gatti
}

Correspondence to:

Professor Gatti.

Received 28 June 1995 Revised version accepted fo publication 6 November 1995

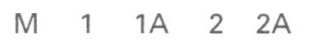

\begin{abstract}
A molecular analysis of the arylsulphatase A gene was performed on 26 unrelated, Italian, late infantile metachromatic leucodystrophy patients. The frequency of the common disease causing mutations $609 \mathrm{~A}$ and $2381 \mathrm{~T}$ was $28 \cdot 8 \%$ and $1.9 \%$ respectively. Pseudodeficiency allele frequency in patients was found to be $13.5 \%$ and a frequency of $10 \cdot 1 \%$ was found in 89 unaffected normal controls. The frequency of the $609 \mathrm{~A}$ mutation in Italian late infantile patients is lower than in late infantile patients from northern Europe, suggesting a higher frequency of different sporadic mutations in the Italian population. A cooperative in cis effect in phenotype determination involving arylsulphatase $A$ mutations and the eventual background of the pseudodeficiency allele is proposed. (f Med Genet 1996;33:251-252)
\end{abstract}

Key words: arylsulphatase A gene; late infantile metachromatic leucodystrophy.

Metachromatic leucodystrophy (MLD) is an autosomal recessively inherited disorder resulting from the deficiency of the lysosomal hydrolase arylsulphatase A (ASA). ${ }^{1}$ Three

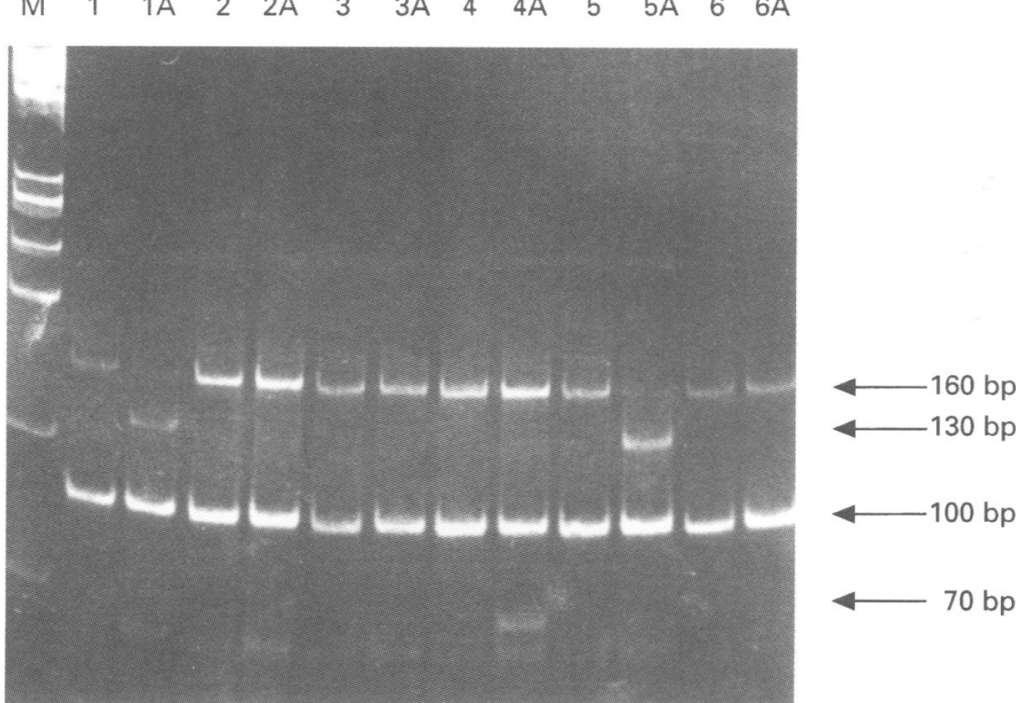

Figure 1 Screening for mutations $609 \mathrm{~A}$ and $2381 \mathrm{~T}$ by a polymerase chain reactionrestriction fragment length polymorphism (PCR-RFLP) method. ${ }^{3}$ It consists of PCR amplification of two fragments of $160 \mathrm{bp}$ and $100 \mathrm{bp}$ containing the sites of mutations $609 A$ and $2381 T$ respectively. In the presence of mutation $609 A$ the $160 \mathrm{bp}$ is XbaI digested into two fragments of $130 \mathrm{bp}$ and $30 \mathrm{bp}$. In the presence of mutation $2381 \mathrm{~T}$ the $100 \mathrm{bp}$ product is PstI digested into two fragments of $70 \mathrm{bp}$ and $30 \mathrm{bp}$. Lane M: $\Phi$ X174 DNA HaelII digested; lanes 1-6: PCR products from six patients; lanes $1 A-6 A$ : XbaI and PstI digested PCR products from the same patients. Patients 1 and 5 are homozygous for the $609 \mathrm{~A}$ mutation; patient 4 is heterozygous for the $2381 \mathrm{~T}$ mutation. different clinical forms of MLD can be distinguished according to the age of onset: a late infantile, a juvenile, and an adult form.

We report the results of screening performed on 26 unrelated, Italian, late infantile MLD patients from different parts of Italy. Patients were investigated for the two most frequent ASA mutations causing MLD: 609A, common in the late infantile phenotype, and $2381 \mathrm{~T}$, common in the adult phenotype (fig 1). ${ }^{23}$ In addition, patients were screened for the pseudodeficiency associated ASA mutations (1788G and $2723 \mathrm{G}$ ) that cause a non-pathological $90 \%$ reduction of ASA activity in homozygous carriers (fig 2). ${ }^{4}$ Pseudodeficiency (pd) allele screening was performed on our patients and on 89 normal controls. Patients were diagnosed by enzymatic assay on cultured fibroblasts. ${ }^{5}$ The clinical form of MLD was determined by the age of onset of symptoms.

The frequency of the 609A mutation was $28.8 \%$ ( 15 alleles out of 52 , fig 3 ), which is lower than the frequency found in other groups of late infantile MLD patients, such as $44.8 \%$ among late infantile MLD patients diagnosed in Germany (26 alleles out of 58 ), ${ }^{6}$ and $55.5 \%$ among late infantile MLD patients diagnosed in Britain (10 alleles out of 18). ${ }^{7}$ This suggests that in Italy there is a higher frequency of rare mutations causing the late infantile phenotype than in northern European populations.

The frequency of mutation $2381 \mathrm{~T}$ among our patients was $1.9 \%$ (one allele out of 52 , fig 3). This low frequency is not surprising, because the $2381 \mathrm{~T}$ mutation is typical of milder phenotypes. The only patient heterozygous for the $2381 \mathrm{~T}$ mutation is a rare case of a late infantile carrier of this mutation. He was also not a carrier of the $609 \mathrm{~A}$ mutation.

Patients and normal controls were also screened for the pd allele (fig 3). Among the patients, the frequency of the pd allele was $13 \cdot 5 \%$ (seven alleles out of 52 ), while among 89 unrelated normal controls the frequency was $10.1 \%$ (18 alleles out of 178 ).

Among the patients who carry the 609A mutation, only one 609A heterozygote is also heterozygous for the pd allele. The only patient carrier of the $2381 \mathrm{~T}$ mutation is also heterozygous for the pd allele. Normal and pd related PCR products obtained with specific oligonucleotides ${ }^{4}$ from this last patient were used for subsequent screening for mutation $2381 \mathrm{~T}^{3}$ Results obtained showed that mutation 2381T and pd associated mutations lie on the same allele in the patient described (data not shown). 
$\begin{array}{lllllllllllllll}M & 1 & 1 \mathrm{~A} & 2 & 2 \mathrm{~A} & 3 & 3 \mathrm{~A} & 4 & 4 \mathrm{~A} & 5 & 5 \mathrm{~A} & 6 & 6 \mathrm{~A} & 7 & 7 \mathrm{~A}\end{array}$

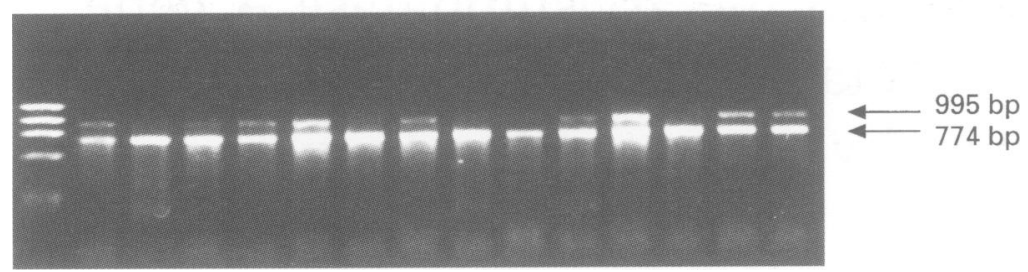

Figure 2 Screening for the pd allele according to a differential amplification method ${ }^{4}$ It consists of separate amplifications of the normal and the pd allele with specific oligonucleotides. In both cases, PCR products are 995 bp long; each normal or pd allele specific amplification is coupled to the amplification of a pd independent control fragment of $774 \mathrm{bp}$. Lane M: $\Phi$ X174 DNA HaeIII digested; lanes 1-7: amplification with normal allele specific oligonucleotides performed on seven patients; lanes $1 A-7 A$ : amplifications with pd allele specific oligonucleotides performed on the same seven patients. Patient 5 is homozygous for the pd allele; patients 2 and 7 are heterozygous for the pd allele; no pd alleles are present in patients 1, 3, 4, 6.

26 MLD patients

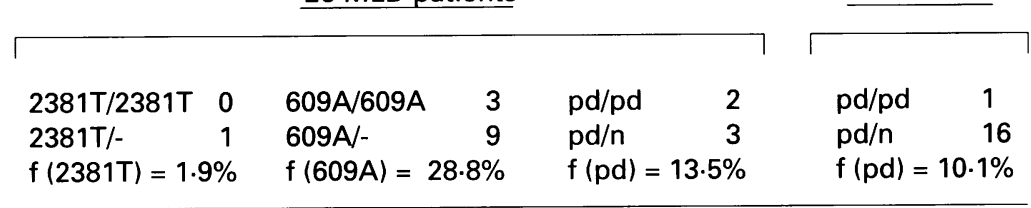

Figure 3 Results of screening for mutations 609A, 2381T, and pd associated ASA mutations performed on 26 unrelated, Italian, late infantile MLD patients and for pd mutations performed on 89 unrelated, Italian, normal controls. Patients and normal controls homozygous and heterozygous for the screened mutations and allelic frequencies are indicated. - is an unknown MLD causing $A S A$ mutation. $n$ is a non-pd allele.

$\mathrm{Pd}$ allele frequencies found among patients and healthy controls $(13.5 \%$ and $10 \cdot 1 \%$ respectively) are in the range found in other populations $(7 \cdot 3 \%$ to $15 \%),{ }^{8}$ but the higher frequency found among patients suggests that it could be possible that certain mutations have more severe consequences if located on a pd allele than if located on a non-pd allele. With a similar cooperative in cis effect, the late infantile phenotype of the above mentioned patient carrier of the $2381 \mathrm{~T}$ mutation on a pd allele could be explained. At present, however, we cannot exclude that an additional mutation is present in this allele and is effectively responsible for the observed phenotype. The high frequency of the pseudodeficiency in the general population and the existence of MLD causing mutations on pd alleles illustrate the problems associated with diagnosis of MLD, which needs a multiple approach, biochemical and molecular, to differentiate between MLD and pd associated ASA deficiencies.

This work was supported by Italian Telethon grants A.25 and C.02/1993.

1 Kolodny EH, Fluharty AL. Metachromatic leukodystrophy and multiple sulfatase deficiency: sulfatide lipidosis. In: Scriver CR, Beaudet AL, Sly WS, Valle D, eds. The metabolic and molecular bases of inherited disease. 7th ed. New York:
McGraw-Hill, 1994:2693-739.

2 Gieselmann V, Zlotogora J, Harris A, Wenger DA, Morris CP. Molecular genetics of metachromatic leukodystrophy. Hum Mutat 1994;4:233-42.

3 Berger J, Molzer B, Gieselmann V, Bernheimer H. Simultaneous detection of the two most frequent metachromatic leukodystrophy mutations. Hum Genet 1993; 92:421-3

4 Gieselmann V. An assay for the rapid detection of the arylsulfatase A pseudodeficiency allele facilitates diagnosis and genetic counseling for metachromic leukodystrophy. Hum Genet 1991;86:251-5.

5 Baum H, Dogson KS, Spencer B. The assay of arylsulfatase A and B in human urine. Clin Chim Acta 1959;4:453-5.

6 Polten A, Fluharty AL, Fluharty CB, Kappler J, von Figura K, Gieselmann V. Molecular basis of different forms of metachromatic leukodystrophy. N Engl f Med 1991;324: 18-22.

7 Barth ML, Fensom A, Harris A. Prevalence of common mutations in the arylfulatase A gene in metachromatic mutations in the arylfulatase A gene in metachromatic leukodystrophy pa

8 Thomas GH. "Pseudodeficiencies" of lysosomal hydrolases. Am $\mathcal{F}$ Hum Genet 1994;54:934-40. 